\title{
Host-ectoparasite Associations: The Role of Host Traits, Season and Habitat on Parasitism Interactions of the Rodents of Northeastern Iran
}

\author{
Kordiyeh Hamidi ( $\nabla$ kordiyeh.hamidi@yahoo.com ) \\ Ferdowsi University of Mashhad https://orcid.org/0000-0003-3206-5258 \\ Rubén Bueno-Marí \\ Universitat de Valencia
}

\section{Research}

Keywords: Parasitic relationship, Rodentia, Host choice, Infestation rate, Prevalence, Zoonotic diseases

Posted Date: July 20th, 2020

DOI: https://doi.org/10.21203/rs.3.rs-42804/v1

License: () (i) This work is licensed under a Creative Commons Attribution 4.0 International License.

Read Full License

Version of Record: A version of this preprint was published on April 1st, 2021. See the published version at https://doi.org/10.1016/j.aspen.2020.12.009. 


\section{Abstract \\ Background}

Rodents play a significant role as reservoirs of zoonotic diseases. Nevertheless, their ectoparasite assemblage and host-ectoparasite associations are poorly known. This study intended to give new insights on the relationships between ectoparasites and rodents in northeastern Iran.

\section{Methods}

Rodents were captured using live traps during the year of 2016-2020 and their ectoparasites were collected. Parasitological indices such as infestation rate, prevalence and mean intensity of infestation were analyzed.

\section{Results}

A total of 284 rodents, belonging to 17 species, were trapped which infested by 178 ectoparasites from five orders Siphonaptera, Phthiraptera, Ixodida, Mesostigmata and Trombidiformes. Overall infestation rate was $50.3 \%$. Flea Nosopsyllus fasciatus and louse Polyplax asiatica were dominated among all fleas and lice, respectively. Haemaphysalis punctata and Haemolaelaps sp. were recorded as the most abundant tick and mite, respectively. Nosopsyllus fasciatus exhibited low and Polyplax asiatica moderate host specificity. Around $64.2 \%$ of ectoparasites shared more than one host and others were singletons. Seasonal fluctuations were found in the occurrence of ectoparasite; fleas and lice were more abundant in spring and winter, respectively. Ticks demonstrated high abundance in spring and summer and mites were more common in autumn. Overall prevalence of ectoparasite on male rodents was greater than females ( $56.4 \%$ vs. $44.4 \%)$, while similar mean intensity were detected for both sexes.

\section{Conclusions}

This study extend the knowledge on the distribution, seasonality and host choice of four main groups of ectoparasites in associations with rodents. Further studies are needed to can provide deep insight into how relationships and interactions between ectoparasite and rodents are formed, and how they can be applied in epidemiology.

\section{Background}

Rodents are considered as the vast majority of the species acting as reservoirs of zoonotic diseases and play a significant role in maintaining ecosystem functionality as seed dispersal agents and arthropod control [1]. Humans with their livestock and pets live in close contact with rodents, exposing them to some zoonotic agents which can be spread to them by direct routes (e.g. through bites or contaminated food 
products or water) or indirectly with parasitic arthropods serving as vectors [2]. Rodents can carry many different species of insects and arachnids, mainly including fleas, lice, ticks, mites, and chiggers [3].

Climate change, urbanization, agriculture, deforestation, habitat degradation and other similar changes in landscape use and species richness leading to biodiversity loss which may increase the abundance of species that thrive in urban areas and the risk of contact with zoonotic pathogens for humans [4]. In other words, the abundance of ectoparasites strongly depends on the abundance of particular rodent species and available host community [5].

In this regard, host-ectoparasite associations provide useful data in order to manage sanitation programs and understand associated epidemiological risks. Host characteristics such as sex, body size, nesting/roosting ecology, and social system can affect ectoparasite diversity or population structure (e.g. $[1,2,6-8])$. Ectoparasites may even synchronize their reproductive cycle in order to access the most profitable host individuals or populations or use propagation strategies to increase their chances to find new hosts [9].

Gender differences in ectoparasite infestation have been reported for a variety of host-ectoparasite systems [10]. Generally, males of higher vertebrates such as mammals are infested by more ectoparasites than females [10-13]. Male-biased parasitism is a complicated phenomenon that is related to: a) difference in mobility and home range size; males of promiscuous or polygynous mammals are more mobile, especially during breeding season, and have larger home ranges and higher spatial overlap between individuals than females. This allows them to increase their mating chances, although it also increases their exposure to parasitism and chances to exchange parasites which resulted in increasing mean species richness of parasite assemblages. In contrast, females generally occupy a separate burrow for parturition due to their territorial individualization, which decreases competition with other females and increases the amount of food per female in the reproductive period $[14,15]$. b) Differences in the immunocompetence between males and females because of the immunosuppressive effect of androgens which fluctuate seasonally being the highest during high reproductive activity and/or selection for androgen-dependent traits due to mate competition [16]. Ectoparasites are expected to influence body condition, due to the energy cost of the immune functions and parasite feeding behaviors [17]. c) Consequently, gender differences in the ectoparasite infestation parameters vary seasonally [16]. However, the seasonal pattern of sex-biased parasitism is poorly known. Last, d) sex-biased parasitism has been also related to sexual size dimorphism; individuals of a larger sex with a smaller body surface/body mass ratio can afford more parasites per unit surface area than individuals of a smaller sex and, thus, should be more protected [18].

Moreover, the distribution of ectoparasites is influenced by the diversity and structure of host communities in different environmental settings [19] as well as hosts' ecology and microhabitat. Especially when habitats are disturbed, resulting in the change of the host assemblage, ectoparasites may encounter other hosts [20]. This can influence ectoparasite dynamics and the transmission of diseases. 
Northeast of Iran has been described as a biodiversity hotspot containing high concentrations of endemic species including terrestrial small mammals (mostly rodents) [21, 22]. As mentioned above, rodents are known as reservoirs for several zoonotic pathogens affecting wild or domestic animals and also humans. While there are many studies on the incidental host or occurrence records of ectoparasites on rodents, mainly in relation to medical and veterinary importance (e.g. [3, 23-25]), relevant knowledge of the ecology and host-ectoparasite relationships, including data on distribution, seasonality or host choice is scarce (e.g. [26-30]).

\section{Methods}

This study aims to contribute to a better understanding on the relationships between ectoparasites and rodents in northeastern parts of Iran. Herein, we 1) provide information on ectoparasite fauna parasitizing rodents (host selection), 2) estimate infestation patterns in rodents species inhabiting different locations (spatial distribution), 3) investigate the seasonality of occurrence of ectoparasites on rodents (temporal distribution), and 4) evaluate the existence of male or female sex-biased parasitism.

\section{Study area}

The study area is located in the northeastern corner of $\operatorname{Iran}\left(37.4761^{\circ} \mathrm{N} 59.6057^{\circ} \mathrm{E}\right)$ which covers an area of $299,231 \mathrm{~km}^{2}$ and has mainly hot dry desert and cold semi-desert climates, and also scattered Mediterranean with spring rains and cold mountains climatic conditions (following [31]). The warmest month (with the highest average high temperature) is July $\left(34.1^{\circ} \mathrm{C}\right.$ ) and the coldest month (with the lowest average low temperature) is January $\left(-3.1^{\circ} \mathrm{C}\right)$. The wettest month (with the highest rainfall) is March (44.3 mm), while the driest month (with the lowest rainfall) is August $(2.2 \mathrm{~mm})$ [32, 33]. Sampling was carried out in 37 localities in northeastern Iran, with five locations in North Khorasan province, 26 locations in Razavi Khorasan province and six in South Khorasan province from April 2016 until May 2020 (Fig. 1).

The study sites mostly consist of forest steppe and semi-desert biotopes, with ten major types of ecological habitat, namely, rocky areas, sandy soils, semi-deserts, meadows, grasslands, woodlands, forests, farms and gardens, parks, and public areas. Northeast of Iran is distributed in Iran-o-Turanian ecological zone. The dominant vegetation consists of Artimisia herbalba, Zygophyllum atriplicoides, Pteropyrum aucheri, Alhagi camelorum, Halocnemum strobilaceum, Aeluropus littoralis and Haloxylon ammodenderon in plain areas, and Amigdallus scoparia, Onobrychis cornuta, Artemisia aucheri, Brumus tumentellus, Acantholimon spp., Astragalus spp. and Alleum spp. in mountain zones [34]. These sites were selected for their suitable habitats for target rodent species, in which we had previously captured the animal [22].

\section{Rodent trapping and collecting of ectoparasites}

Rodents were captured using custom-made live traps during several continuous periods of 12 months. Surveys were conducted during 66 short-term (two days) rodent trapping filed works. A total of 30 traps 
were used for each trapping session, which resulted in 3958 trap-nights (= the number of traps set multiplied by the number of nights deployed, minus number of misfires and non-target species [35]. Traps baited with scorched sunflower seeds, gourd seeds and walnut, were exposed for a day (one trapping session) and checked in the early morning to avoid death of trapped animal and subsequent loss of ectoparasites. Traps were placed on the ground close to burrows, and along the existing trails and corridors (Fig. 2). On two occasions, two non-target animals (Lagomorpha: Afghan pika Ochotona rufescens and Erinaceomorpha: Long-eared hedgehog Hemiechinus auritus) were caught, which were excluded from further data analyses.

After removal from the trap, rodents were individually stored in cotton bags, until processing. Ectoparasites were collected from live-captured rodents. Thus, the body surface of captured rodents was systematically checked for ectoparasites by combing with a fine tooth comb over a white plastic pan and picking carefully using fine forceps. The ectoparasites found in each individual were preserved in $96 \%$ ethanol and stored in labelled individual vials. All aspects of protocols for collection and processing were designed to minimize the likelihood of contamination (i.e. the assignment of ectoparasites to the wrong host individual). Hence, also for dead captured animals, each individual host was placed in a separate clean marked plastic bags before collecting ectoparsites to prevent contamination of ectoparasites between different host individuals.

All trapped rodents were later identified up to species level using available morphological keys and published taxonomic references [36]. Then, sexed, weighed (to $\pm 1 \mathrm{~g}$ ) and their age recorded (based on several signs such as weight, color of the dorsal body fur, status of genital system and eminency of nipple glands, development of the tail tuft, and tooth-wear if needed). After ectoparasite removal, each rodent was released at its trapping point. The animals which were found dead in the traps (only 11 specimens), were transferred to the laboratory for further taxonomic studies on their skull and teeth. Trapping and animal care was performed in compliance with the "Guidelines for the care and use of laboratory and experimental animals", Rodentology Research Group, Ferdowsi University of Mashhad [37].

Dark color ectoparasite specimens (fleas, ticks and some lice) were clarified in potassium hydroxide and then treated with Nesbitt's fluid. Subsequently, mounted on Hoyer liquid, and examined under a stereoscope. Other collected specimens (mainly mites) were directly mounted on glass slides using Hoyer's medium. Ectoparasites species were identified morphologically using taxonomic keys, and systematic manuals and drawings (e.g. [25, 38-42]) (Figs. 3, 4).

\section{Data analyses; Calculation of parasitological parameters}

Host infestation by each group of ectoparasite was described using the following parasitological indices (following [43-45]):

1) Infestation rate is calculated as the number of rodents infested by ectoparasite(s) divided by number of all captured rodents multiplied by 100 . Infestation rate for each ectoparasite group is the number of rodents infested by each ectoparasite group divided by number of rodents infested by all groups multiply 
100. 2) Prevalence of infestation: the percentage of hosts carrying each group of ectoparasite or species. Last, 3) mean intensity of the infection: the mean number of each group of ectoparasite or species per infected host, respectively. Data analyses were performed in SPSS statistics software v. 16.0 for Windows (SPSS Inc, 2007, Chicago, IL, USA).

\section{Results}

During this study, 284 individuals of rodents belonging to 17 species were trapped in 38 locations distributed in northeast of Iran, including Apodemus witherbeyi, Mus musculus, Nesokia indica, Rattus norvegicus, Rattus pytoris, Meriones libycus, Meriones persicus, Rhombomys opimus, and Tatera indica, (Muridae), Cricetulus migratorius, Ellobius fuscocapillus, Microtus paradoxus, and Microtus transcaspicus (Cricetidae), Calomyscus elburzensis and Calomyscus hotsoni (Calomyscidae), Scaratus elater (Dipodidae), and Spermophilus fulvus (Sciuridae). The highest capture success was recorded for Meriones persicus (43 out of 284: 15.1\%) followed by Mus musculus (30 out of 284: 10.5\%), while the lowest capture success was recorded for Rhombomys opimus and Rattus pyctoris both with $0.3 \%$ (1 out of 284 ).

The highest number of captured rodents was recorded from Sangbast (Razavi Khorasan: Mashhad-Torbat road) (39 specimens out of 284: 13.7\%), and the lowest number was recorded from Robat-e mahi (Razavi Khorasan: Sarakhs) and Kahoo and Golbahar (Razavi Khorasan), all with $0.3 \%$ (1 specimens out of 284). Although, the number of rodent species (diversity of hosts) in Sangbast and Soltanabad (Razavi Khorasan: Chenaran) was higher than in the rest of sampling sites (6 out of 17 rodent species: $35.2 \%$ ). In contrast, Baba Ramezan (Kalat), Chenaran, Firouzabad (Mashhad), Golbahar, Kahoo, Robat-e mahi, Tirgan (Dargaz) in Razavi Khorasan and Qaen, Sarayan, and Shadan and Olang heights (Birjand) in South Khorasan showed the lowest number and diversity in the captured rodent's species with only one captured species (5.8\%).

The rocky areas were strongly dominated by Meriones persicus and public areas by Mus musculus and Rattus norvegicus. Ellobius fuscocapillus and Microtus transcaspicus were dominant captured species in the grasslands and parks, respectively, while the meadows and sandy soils were both dominated by Tatera indica and Spermophilus fulvus.

Only one individuals (out of 284: $0.3 \%$ ) was infested with all four groups of ectoparasitic. Moreover, four $(1.4 \%)$ and five $(1.7 \%)$ individuals harboring three and two groups of ectoparasites, respectively. A total of 275 individuals (96.8\%) were parasitized by only one ectoparasite group. Hence, among the infested animals, 133 out of 143 (93\%) specimens of rodents were noted to carry only one group of ectoparasites, while only 10 out of 143 (approximately 7\%) specimens of rodents were noted to carry more than one group of ectoparasites.

Overall infestation rate was $50.3 \%$ (143 out of 284 ). The infestation rate with fleas, lice, ticks and mites were $58.7 \%, 30 \%, 13.2 \%$ and $9 \%$, respectively. Fleas with five known and three unknown species, belonging to three genera, were the most diverse and prevalent group (29.5\%), followed by lice (15.1\%), while the 
lowest prevalence was observed in the mites group (4.5\%), and followed by ticks (6.6\%). Ticks group with two genera, including two known and two unknown species, showed the lowest diversity (Table 1). 
Table 1

List of collected ectoparasites parasitized rodents captured from northeastern Iran

\begin{tabular}{|c|c|c|}
\hline Order & Family & Species \\
\hline \multirow[t]{13}{*}{ Siphonaptera (fleas) } & Ceratophyllidae & Ctenophthalmus pseudagyrtes \\
\hline & \multirow[t]{7}{*}{ Dampf, 1908} & Baker, 1904 \\
\hline & & Ctenophthalmus sp. \\
\hline & & Nosopsyllus fasciatus \\
\hline & & (Bosc, 1801) \\
\hline & & Nosopsyllus iranus \\
\hline & & Wagner and Argyropulo, 1934 \\
\hline & & Nosopsyllus sp. \\
\hline & \multirow{5}{*}{$\begin{array}{l}\text { Pulicidae } \\
\text { Billberg, } 1820\end{array}$} & Xenopsylla buxtoni \\
\hline & & Jordan, 1949 \\
\hline & & Xenopsylla cheopis \\
\hline & & (Rothschild, 1903) \\
\hline & & Xenopsylla sp. \\
\hline \multirow[t]{10}{*}{ Phthiraptera (lice) } & Hoplopleuridae & Hoplopleura captiosa \\
\hline & Ewing, 1929 & Johnson, 1960 \\
\hline & Polyplacidae & Polyplax asiatica \\
\hline & \multirow[t]{7}{*}{ Fahrenholz, 1912} & Ferris, 1923 \\
\hline & & Polyplax gerbilli \\
\hline & & Ferris, 1923 \\
\hline & & Polyplax paradoxa \\
\hline & & (Johnson, 1960) \\
\hline & & Polyplax spinulosa \\
\hline & & (Burmeister, 1839) \\
\hline \multirow[t]{3}{*}{ Ixodida (ticks) } & Ixodidae & Haemaphysalis punctata \\
\hline & \multirow[t]{2}{*}{ C. L. Koch, 1844} & Canestrini and Fanzago, 1878 \\
\hline & & Haemaphysalis sp. \\
\hline
\end{tabular}




\begin{tabular}{|lll|}
\hline Order & Family & Species \\
\hline & & Ixodes trianguliceps \\
& & Birula, 1895 \\
\cline { 2 - 3 } Mesostigmata (mites) & Hirstionyssidae & Ixodes sp. \\
& Evans and Till, 1966 & Zemskaja, 1955 \\
\cline { 2 - 3 } & Laelapidae & Haemolaelaps sp. \\
& Berlese, 1892 & Laelaps algericus \\
& & Hirst, 1925 \\
& & Laelaps sp. \\
\hline Trombidiformes (mites) & Trombiculidae & Microtrombicula sp. \\
\cline { 2 - 3 } & Ewing, 1929 & \\
\hline
\end{tabular}

Spermophilus fulvus showed the highest mean intensity (0.52), followed by Nesokia indica (0.37). Mean intensity about 0.3 was recorded for three species Cricetulus migratorius, Meriones persicus and Mus musculus. Due to showing no infestation, the lowest intensity with the value of zero belonged to Calomyscus hotsoni and Scaratus elater. Other rodent species including Apodemus witherbeyi, Calomyscus elburzensis, Ellobius fuscocapillus, Meriones libycus, Microtus paradoxus, Microtus transcaspicus, Rattus norvegicus, Rattus pyctoris, Rhombomys opimus, and Tatera indica showed intensity value of 0.25 . Detailed information is presented in Table 2. 
Table 2

List of captured rodents together with their ectoparasite assemblage. For each host species, the numbers of captured and overall infested individuals are provided in parenthesis, respectively

\begin{tabular}{|c|c|c|c|c|}
\hline Host Species & Parasitic flea & $\begin{array}{l}\text { Parasitic } \\
\text { louse }\end{array}$ & Parasitic tick & Parasitic mite \\
\hline $\begin{array}{l}\text { Apodemus } \\
\text { witherbeyi } \\
(19,7)\end{array}$ & - & - & $\begin{array}{l}\text { Haemaphysalis } \\
\text { sp., Ixodes sp. }\end{array}$ & - \\
\hline $\begin{array}{l}\text { Calomyscus } \\
\text { elburzensis } \\
(4,4)\end{array}$ & Nosopsyllus iranus & - & - & - \\
\hline $\begin{array}{l}\text { Calomyscus } \\
\text { hotsoni }(7,0)\end{array}$ & - & - & - & - \\
\hline $\begin{array}{l}\text { Cricetulus } \\
\text { migratorius } \\
(24,9)\end{array}$ & Nosopsyllus iranus & $\begin{array}{l}\text { Polyplax } \\
\text { asiatica }\end{array}$ & - & - \\
\hline $\begin{array}{l}\text { Ellobius } \\
\text { fuscocapillus } \\
(23,4)\end{array}$ & - & - & - & $\begin{array}{l}\text { Haemolaelaps } \\
\text { sp. }\end{array}$ \\
\hline $\begin{array}{l}\text { Meriones } \\
\text { libycus }(17,7)\end{array}$ & Nosopsyllus fasciatus & - & - & $\begin{array}{l}\text { Hirstionyssus } \\
\text { sp. }\end{array}$ \\
\hline $\begin{array}{l}\text { Meriones } \\
\text { persicus (43, } \\
33)\end{array}$ & $\begin{array}{l}\text { Nosopsylla fasciatus, } \\
\text { Nosopsyllus iranus, } \\
\text { Xenopsylla buxtoni, } \\
\text { Xenopsylla cheopis }\end{array}$ & $\begin{array}{l}\text { Polyplax } \\
\text { asiatica, } \\
\text { Polyplax } \\
\text { paradoxa }\end{array}$ & $\begin{array}{l}\text { Ixodes } \\
\text { trianguliceps, } \\
\text { Haemaphysalis } \\
\text { punctata }\end{array}$ & $\begin{array}{l}\text { Haemolaelaps } \\
\text { sp. }\end{array}$ \\
\hline $\begin{array}{l}\text { Microtus } \\
\text { paradoxus (3, } \\
\text { 3) }\end{array}$ & Nosopsyllus fasciatus & - & - & - \\
\hline $\begin{array}{l}\text { Microtus } \\
\text { transcaspicus } \\
(20,8)\end{array}$ & $\begin{array}{l}\text { Nosopsyllus fasciatus, } \\
\text { Xenopsylla buxtoni }\end{array}$ & - & - & - \\
\hline $\begin{array}{l}\text { Mus } \\
\text { musculus }(30, \\
16)\end{array}$ & $\begin{array}{l}\text { Ctenophthalmus sp., } \\
\text { Nosopsyllus fasciatus, } \\
\text { Nosopsyllus iranus }\end{array}$ & $\begin{array}{l}\text { Hoplopleura } \\
\text { captiosa }\end{array}$ & $\begin{array}{l}\text { Haemaphysalis } \\
\text { punctata, } \\
\text { Haemaphysalis } \\
\text { sp. }\end{array}$ & $\begin{array}{l}\text { Haemolaelaps } \\
\text { sp., Laelaps } \\
\text { algericus, } \\
\text { Laelaps sp., } \\
\text { Microtrombicula } \\
\text { sp. }\end{array}$ \\
\hline $\begin{array}{l}\text { Nesokia } \\
\text { indica }(20, \\
16)\end{array}$ & $\begin{array}{l}\text { Nosopsylla fasciatus, } \\
\text { Nosopsylla sp., Xenopsylla } \\
\text { cheopis, Xenopsylla sp. }\end{array}$ & $\begin{array}{l}\text { Hoplopleura } \\
\text { captiosa, } \\
\text { Polyplax } \\
\text { asiatica }\end{array}$ & $\begin{array}{l}\text { Haemaphysalis } \\
\text { punctata }\end{array}$ & - \\
\hline
\end{tabular}




\begin{tabular}{|lllll|}
\hline Host Species & Parasitic flea & $\begin{array}{l}\text { Parasitic } \\
\text { louse }\end{array}$ & Parasitic tick & Parasitic mite \\
\hline $\begin{array}{l}\text { Rattus } \\
\text { norvegicus } \\
(26,15)\end{array}$ & $\begin{array}{l}\text { Ctenophtalmus pseudagyrtes, } \\
\text { Ctenophtalmus sp., } \\
\text { Nosopsyllus fasciatus, } \\
\text { Xenopsylla buxtoni, } \\
\text { Xenopsylla cheopis }\end{array}$ & $\begin{array}{l}\text { Hoplopleura } \\
\text { captiosa, }\end{array}$ & $\begin{array}{l}\text { Polyplax } \\
\text { gerbilli, } \\
\text { Polyplax } \\
\text { spinulosa }\end{array}$ & \\
\hline $\begin{array}{l}\text { Rattus } \\
\text { pyctoris }(1,1)\end{array}$ & Xenopsylla buxtoni & - & - & \\
$\begin{array}{l}\text { Rhombomys } \\
\text { opimus }(1,1)\end{array}$ & Nosopsyllus fasciatus & - & - & \\
\hline $\begin{array}{l}\text { Scaratus } \\
\text { elater }(3,0)\end{array}$ & - & - & - & - \\
\hline $\begin{array}{l}\text { Spermophilus } \\
\text { fulvus }(25,\end{array}$ & Nosopsylla fasciatus & $\begin{array}{l}\text { Polyplax } \\
\text { asiatica }\end{array}$ & - & - \\
\hline $\begin{array}{l}\text { Tatera indica } \\
(18,7)\end{array}$ & Xenopsylla buxtoni & $\begin{array}{l}\text { Polyplax } \\
\text { gerbilli }\end{array}$ & - & - \\
\hline
\end{tabular}

A total of 178 specimens of ectoparasites from five orders Siphonaptera (89 specimens), Phthiraptera (57 specimens), Ixodida (19 specimens), Mesostigmata (12 specimens) and Trombidiformes (1 specimens) were collected and identified from the infested hosts. This can be further categorized into three, two, two and four genera of flea, louse, tick and mite, respectively including Ctenophthalmus pseudagyrtes, Ctenophthalmus sp., Nosopsyllus fasciatus, Nosopsyllus iranus, Nosopsyllus sp., Xenopsylla buxtoni, Xenopsylla cheopis, and Xenopsylla sp. (fleas: Siphonaptera), Hoplopleura captiosa, Polyplax asiatica, Polyplax gerbilli, Polyplax paradoxa, and Polyplax spinulosa (sucking lice: Phthiraptera), Haemaphysalis punctata, Haemaphysalis sp., Ixodes trianguliceps, and Ixodes sp. (ticks: Ixodida), and Haemolaelaps sp., Hirstionyssus meridianus, Laelaps algericus, and Laelaps sp. (mites: Mesostigmata), and Microtrombicula sp. (mites: Trombidiformes) (Table 1).

Of the parasitic fleas and lice, Nosopsyllus fasciatus (Ceratophyllidae) and Polyplax asiatica (Polyplacidae) were dominated (50.5\% and $52.6 \%$ of all fleas and lice, respectively). For tick and mite groups, Haemaphysalis punctata (Ixodidae) (36.84\%) and Haemolaelaps sp. (Laelapidae) (61.5\%) were recorded as the most abundant sampled ectoparasites.

The values of abundance and infestation with ectoparasites groups varied between host species and sampling locations. Mus musculus had the most diverse ectoparasite assemblage (10 ectoparasite species) followed by Meriones persicus (9 ectoparasite species), Rattus norvegicus (8 ectoparasite species) and Nesokia indica (7 ectoparasite species), suggesting that these species can accommodate variety of ectoparasites (Table 2). In contrast, Calomyscus hotsoni and Scaratus elater carried no ectoparasite. 
The two most commonly found ectoparasite species were Nosopsyllus fasciatus (45 out of 178 specimens: $25.2 \%$ ) and Polyplax asiatica (30 out of 178 specimens: $16.8 \%$ ). The flea Nosopsyllus fasciatus exhibited low host specificity, infesting diverse species of rodents (52.9\%: 9 out of 17 rodent species including Meriones libycus, Meriones persicus, Microtus paradoxus, Microtus transcaspicus, Mus musculus, Nesokia indica, Rattus norvegicus, Rhombomys opimus and Spermophilus fulvus). The louse Polyplax asiatica was found on 4 out of 17 rodent species (23.5\%), exhibiting moderate host specificity evident by infesting Cricetulus migratorius, Meriones persicus, Nesokia indica, and Spermophilus fulvus. Among the known species of ectoparasite, the flea Ctenophthalmus pseudagyrtes was only recorded in Rattus norvegicus, and the lice species Polyplax paradoxa and Polyplax spinulosa were only found on Meriones persicus and Rattus norvegicus, respectively. The tick Ixodes trianguliceps was also collected only from Meriones persicus and the mite Laelaps algericus from Mus musculus. Thus, each of these five ectoparasite species showed high host specificity (1 out of 17 rodent species: $5.8 \%$ ) (Table 3 ). Hence, nine known species of ectoparasites (around $64.2 \%$ ) shared more than one host and approximately $35.7 \%$ of them $(n=5$ out of 14$)$ were singletons.

Table 3

Host selection in ectoparasite assemblage collected from northeastern Iran; list of collected ectoparasites showing high host specificity in association with the rodent which they hosted

\begin{tabular}{|lll|}
\hline Ectoparasitic group & Ectoparasite species & Host \\
\hline Flea & Ctenophtalmus pseudagyrtes & Rattus norvegicus \\
\cline { 2 - 3 } & Nosopsylla sp. & Nesokia indica \\
\cline { 2 - 3 } & Xenopsylla sp. & \\
\hline Lice & Polyplax paradoxa & Meriones persicus \\
\hline Tick & Polyplax spinulosa & Rattus norvegicus \\
\hline Mite & Ixodes trianguliceps & Meriones persicus \\
& Laelaps algericus & Apodemus witherbeyi \\
\hline & Laelaps sp. & Mus musculus \\
& Microtrombicula sp. & \\
\hline Hirstionyssus sp. & Meriones libycus \\
\hline
\end{tabular}

Of the parasitic fleas, Nosopsyllus fasciatus was dominant species, found in 19 out of 37 sampling localities (51.3\%). In contrast, Ctenophthalmus pseudagyrtes was only recorded from Faruj in North Khorasan (2.7\%). Among lice, Polyplax asiatica was considered as the most common species (11 out of 37: 29.7\%), while Polyplax spinulosa and Polyplax gerbilli were reported as the rarest one (5.4\%), both were only recorded from Faruj and Mashhad-Torbat road (Razavi Khorasan). Of the tick group, genus 
Haemaphysalis showed dominancy as compared with genus Ixodes (21.6\% vs. $16.2 \%)$, found in eight and six sampling localities, respectively. Last, members of the genus Haemolaelaps was the most common mite (3 out of 37: 8.1\%). In contrast, each of the mite species Laelaps algericus and Hirstionyssus meridianus as well as two unknown species Laelaps sp. and Microtrombicula sp. were only found in one locality $(2.7 \%)$ (Figs. 3, 4).

Species Nosopsyllus fasciatus was recorded as a common flea for most of the sampling habitats (7 out of 10: 70\%), but species Ctenophthalmus pseudagyrtes, found from public areas, was unique for its respective habitat (10\%). Lice species Polyplax asiatica and Hoplopleura captiosa were found in half of the habitat types (50\%), although Polyplax paradoxa (recorded from grassland and rocky areas) and Polyplax spinulosa (reported from meadows and public areas) were the less common lice species, both found in only two habitat types (each with $20 \%$ frequency). Among tick group, genus Ixodes was the most common tick found in four habitat types (40\%). However, Ixodes trianguliceps was only captured from rodents inhabit rocky areas (10\%). Mite genus Laelaps was recorded as the most common mite found in $30 \%$ of habitat types. In contrast, Laelaps algericus, Hirstionyssus meridianus and Microtrombicula sp. were only found in farms and gardens, sandy soils, and public areas, respectively (each with $10 \%$ frequency). Detailed information is presented in Table 4. 
Table 4

Spatial distribution of ectoparasites on rodents trapped in different habitats in northeastern Iran

\begin{tabular}{|c|c|c|c|c|c|}
\hline $\begin{array}{l}\text { Habitat } \\
\text { type }\end{array}$ & Host Species & Parasitic flea & $\begin{array}{l}\text { Parasitic } \\
\text { louse }\end{array}$ & Parasitic tick & Parasitic mite \\
\hline $\begin{array}{l}\text { Farm and } \\
\text { garden }\end{array}$ & $\begin{array}{l}\text { Apodemus } \\
\text { witherbeyi, } \\
\text { Cricetulus } \\
\text { migratorius, } \\
\text { Meriones } \\
\text { libycus, Mus } \\
\text { musculus, } \\
\text { Rattus } \\
\text { norvegicus }\end{array}$ & $\begin{array}{l}\text { Ctenophthalmus } \\
\text { sp., Xenopsylla } \\
\text { buxtoni, } \\
\text { Xenopsylla } \\
\text { cheopis }\end{array}$ & - & Ixodes sp. & $\begin{array}{l}\text { Laelaps } \\
\text { algericus }\end{array}$ \\
\hline Forest & $\begin{array}{l}\text { Calomyscus } \\
\text { hotsoni, Mus } \\
\text { musculus, } \\
\text { Spermophilus } \\
\text { fulvus }\end{array}$ & - & - & - & - \\
\hline Grassland & $\begin{array}{l}\text { Apodemus } \\
\text { witherbeyi, } \\
\text { Cricetulus } \\
\text { migratorius, } \\
\text { Ellobius } \\
\text { fuscocapillus, } \\
\text { Meriones } \\
\text { libycus, } \\
\text { Meriones } \\
\text { persicus, } \\
\text { Microtus } \\
\text { transcaspicus, } \\
\text { Mus } \\
\text { musculus, } \\
\text { Rattus } \\
\text { norvegicus, } \\
\text { Spermophilus } \\
\text { fulvus }\end{array}$ & $\begin{array}{l}\text { Nosopsyllus } \\
\text { fasciatus, } \\
\text { Nosopsyllus } \\
\text { iranus, } \\
\text { Xenopsylla } \\
\text { buxtoni, } \\
\text { Xenopsylla } \\
\text { cheopis }\end{array}$ & $\begin{array}{l}\text { Polyplax } \\
\text { paradoxa }\end{array}$ & Ixodes sp. & $\begin{array}{l}\text { Haemolaelaps } \\
\text { sp. }\end{array}$ \\
\hline Meadow & $\begin{array}{l}\text { Apodemus } \\
\text { witherbeyi, } \\
\text { Ellobius } \\
\text { fuscocapillus, } \\
\text { Meriones } \\
\text { libycus, Mus } \\
\text { musculus, } \\
\text { Nesokia } \\
\text { indica, Rattus } \\
\text { norvegicus, } \\
\text { Rhombomys } \\
\text { opimus, } \\
\text { Spermophilus } \\
\text { fulvus, Tatera } \\
\text { indica }\end{array}$ & $\begin{array}{l}\text { Nosopsyllus } \\
\text { fasciatus, } \\
\text { Nosopsyllus } \\
\text { iranus, } \\
\text { Xenopsylla sp. }\end{array}$ & $\begin{array}{l}\text { Hoplopleura } \\
\text { captiosa, } \\
\text { Polyplax } \\
\text { asiatica, } \\
\text { Polyplax } \\
\text { gerbilli, } \\
\text { Polyplax } \\
\text { spinulosa }\end{array}$ & $\begin{array}{l}\text { Haemaphysalis } \\
\text { sp. }\end{array}$ & - \\
\hline
\end{tabular}




\begin{tabular}{|c|c|c|c|c|c|}
\hline $\begin{array}{l}\text { Habitat } \\
\text { type }\end{array}$ & Host Species & Parasitic flea & $\begin{array}{l}\text { Parasitic } \\
\text { louse }\end{array}$ & Parasitic tick & Parasitic mite \\
\hline Park & $\begin{array}{l}\text { Ellobius } \\
\text { fuscocapillus, } \\
\text { Microtus } \\
\text { transcaspicus, } \\
\text { Mus } \\
\text { musculus, } \\
\text { Nesokia } \\
\text { indica }\end{array}$ & $\begin{array}{l}\text { Nosopsyllus } \\
\text { fasciatus }\end{array}$ & $\begin{array}{l}\text { Hoplopleura } \\
\text { captiosa, } \\
\text { Polyplax } \\
\text { asiatica }\end{array}$ & - & $\begin{array}{l}\text { Haemolaelaps } \\
\text { sp., Laelaps sp. }\end{array}$ \\
\hline $\begin{array}{l}\text { Public } \\
\text { area }\end{array}$ & $\begin{array}{l}\text { Meriones } \\
\text { libycus, Mus } \\
\text { musculus, } \\
\text { Nesokia } \\
\text { indica, Rattus } \\
\text { norvegicus, } \\
\text { Spermophilus } \\
\text { fulvus }\end{array}$ & $\begin{array}{l}\text { Ctenophtalmus } \\
\text { pseudagyrtes, } \\
\text { Nosopsylla } \\
\text { fasciatus, } \\
\text { Nosopsyllus } \\
\text { iranus, } \\
\text { Xenopsylla } \\
\text { buxtoni, } \\
\text { Xenopsylla } \\
\text { cheopis }\end{array}$ & $\begin{array}{l}\text { Hoplopleura } \\
\text { captiosa, } \\
\text { Polyplax } \\
\text { asiatica, } \\
\text { Polyplax } \\
\text { spinulosa }\end{array}$ & $\begin{array}{l}\text { Haemaphysalis } \\
\text { punctata }\end{array}$ & $\begin{array}{l}\text { Haemolaelaps } \\
\text { sp., Laelaps sp., } \\
\text { Microtrombicula } \\
\text { sp. }\end{array}$ \\
\hline $\begin{array}{l}\text { Rocky } \\
\text { area }\end{array}$ & $\begin{array}{l}\text { Apodemus } \\
\text { witherbeyi, } \\
\text { Calomyscus } \\
\text { elburzensis, } \\
\text { Calomyscus } \\
\text { hotsoni, } \\
\text { Cricetulus } \\
\text { migratorius, } \\
\text { Meriones } \\
\text { libycus, } \\
\text { Meriones } \\
\text { persicus, } \\
\text { Microtus } \\
\text { paradoxus, } \\
\text { Microtus } \\
\text { transcaspicus, } \\
\text { Mus } \\
\text { musculus, } \\
\text { Nesokia } \\
\text { indica, Rattus } \\
\text { norvegicus, } \\
\text { Rattus } \\
\text { pyctoris }\end{array}$ & $\begin{array}{l}\text { Ctenophtalmus } \\
\text { sp., Nosopsyllus } \\
\text { fasciatus, } \\
\text { Nosopsyllus } \\
\text { iranus, } \\
\text { Xenopsylla } \\
\text { buxtoni, } \\
\text { Xenopsylla } \\
\text { cheopis }\end{array}$ & $\begin{array}{l}\text { Hoplopleura } \\
\text { captiosa, } \\
\text { Polyplax } \\
\text { asiatica, } \\
\text { Polyplax } \\
\text { paradoxa }\end{array}$ & $\begin{array}{l}\text { Haemaphysalis } \\
\text { punctata, } \\
\text { Haemaphysalis } \\
\text { sp., Ixodes } \\
\text { trianguliceps, } \\
\text { Ixodes sp. }\end{array}$ & $\begin{array}{l}\text { Haemolaelaps } \\
\text { sp. }\end{array}$ \\
\hline $\begin{array}{l}\text { Sandy } \\
\text { soil }\end{array}$ & $\begin{array}{l}\text { Meriones } \\
\text { libycus, Mus } \\
\text { musculus, } \\
\text { Nesokia } \\
\text { indica, } \\
\text { Scaratus } \\
\text { elater, } \\
\text { Spermophilus } \\
\text { fulvus, Tatera } \\
\text { indica }\end{array}$ & $\begin{array}{l}\text { Nosopsylla } \\
\text { fasciatus, } \\
\text { Nosopsyllus } \\
\text { iranus, } \\
\text { Nosopsylla sp., } \\
\text { Xenopsylla } \\
\text { buxtoni }\end{array}$ & $\begin{array}{l}\text { Hoplopleura } \\
\text { captiosa, } \\
\text { Polyplax } \\
\text { asiatica, } \\
\text { Polyplax } \\
\text { gerbilli }\end{array}$ & $\begin{array}{l}\text { Haemaphysalis } \\
\text { punctata, } \\
\text { Haemaphysalis } \\
\text { sp. }\end{array}$ & $\begin{array}{l}\text { Hirstionyssus } \\
\text { sp. }\end{array}$ \\
\hline
\end{tabular}




\begin{tabular}{|llllll|}
\hline $\begin{array}{l}\text { Habitat } \\
\text { type }\end{array}$ & Host Species & Parasitic flea & $\begin{array}{l}\text { Parasitic } \\
\text { louse }\end{array}$ & Parasitic tick & Parasitic mite \\
\hline $\begin{array}{l}\text { Semi- } \\
\text { desert }\end{array}$ & $\begin{array}{l}\text { Meriones } \\
\text { libycus, Tatera } \\
\text { indica }\end{array}$ & $\begin{array}{l}\text { Xenopsylla } \\
\text { buxtoni }\end{array}$ & $\begin{array}{l}\text { Polyplax } \\
\text { gerbilli }\end{array}$ & - & - \\
\hline & $\begin{array}{l}\text { Apodemus } \\
\text { Witherbeyi, } \\
\text { Ellobius } \\
\text { fuscocapillus, } \\
\text { Meriones } \\
\text { persicus, Mus } \\
\text { musculus }\end{array}$ & $\begin{array}{l}\text { Nosopsyllus } \\
\text { fasciatus }\end{array}$ & - & Ixodes sp. & - \\
\hline
\end{tabular}

\section{Parasitism interaction and seasonality}

Seasonal fluctuations were found in the occurrence of ectoparasite, with remarkable differences according to flea species; fleas were more abundant in spring (50 collected flea specimens in springs out of 89 in other seasons: $56.1 \%$ ). Lice were more common in winter (19 out of $57: 33.3 \%$ ), and ticks demonstrated high abundance with equal value in spring and summer ( 6 out of 19:31.5\%) decreasing in numbers as the season progressed. Last, mites showed a high abundance in the autumn (7 out of 13: 53.8\%). Fleas, lice and ticks were recorded in all sampling months, while members of mite group had no record in the summer.

Flea species Nosopsyllus fasciatus was reported with most records in all sampling months (mean intensity $0.38,0.68,0.66$, and 0.76 in spring, summer, autumn, and winter, respectively). Louse species Polyplax asiatica showed the highest mean intensity among other lice species in spring (0.85), summer (0.38), and winter (1), while Hoplopleura captiosa was recorded as the most common lice species in the autumn (0.6). Tick species Haemaphysalis punctata was more common in spring and autumn (mean intensity 0.5 and 0.75 , respectively), while Ixodes sp. and Ixodes trianguliceps showed the highest mean intensity (with value of 0.66 for each one) in the summer and winter, respectively. Finally, mite Haemolaelaps sp. with most records in the spring and autumn showed mean intensity 0.75 and 0.71 , respectively, while species Hirstionyssus meridianus, which was recorded exclusively in the winter, along with Laelaps sp. were the most common lice in winter, each with mean intensity 0.5 (Table 5). 
Table 5

Temporal (seasonal) distribution of ectoparasites parasitized rodents in northeastern Iran. The number given in parenthesis show the number of collected ectoparasite

\begin{tabular}{|c|c|c|c|c|}
\hline $\begin{array}{l}\text { Capturing } \\
\text { season }\end{array}$ & Parasitic flea & Parasitic louse & Parasitic tick & Parasitic mite \\
\hline \multirow[t]{2}{*}{ Spring } & $\begin{array}{l}\text { Ctenophthalmus sp. (1), } \\
\text { Nosopsyllus fasciatus } \\
\text { (18), Nosopsyllus iranus } \\
\text { (12), Xenopsylla buxtoni } \\
\text { (6), Xenopsylla cheopis } \\
\text { (13) }\end{array}$ & $\begin{array}{l}\text { Hoplopleura captiosa } \\
\text { (1), Polyplax asiatica } \\
(12), \text { Polyplax } \\
\text { paradoxa (4) }\end{array}$ & $\begin{array}{l}\text { Ixodes } \\
\text { trianguliceps } \\
(1), \\
\text { Haemaphysalis } \\
\text { punctata (3), } \\
\text { Haemaphysalis } \\
\text { sp. (2) }\end{array}$ & $\begin{array}{l}\text { Haemolaelaps } \\
\text { sp. (3), Laelaps } \\
\text { algericus (1) }\end{array}$ \\
\hline & $\begin{array}{l}\text { No. of rodents infected } \\
\text { by flea: } 47\end{array}$ & $\begin{array}{l}\text { No. of rodents } \\
\text { infected by louse: } 14\end{array}$ & $\begin{array}{l}\text { No. of rodents } \\
\text { infected by } \\
\text { tick: } 6\end{array}$ & $\begin{array}{l}\text { No. of rodents } \\
\text { infected by } \\
\text { mite: } 4\end{array}$ \\
\hline \multirow[t]{2}{*}{ Summer } & $\begin{array}{l}\text { Ctenophtalmus } \\
\text { pseudagyrtes (1), } \\
\text { Ctenophtalmus sp. (1), } \\
\text { Nosopsyllus fasciatus } \\
\text { (11), Nosopsyllus iranus } \\
\text { (2), Xenopsylla buxtoni } \\
\text { (2) }\end{array}$ & $\begin{array}{l}\text { Hoplopleura captiosa } \\
(2), \text { Polyplax asiatica } \\
(5), \text { Polyplax gerbilli } \\
(4), \text { Polyplax } \\
\text { paradoxa (3), } \\
\text { Polyplax spinulosa } \\
\text { (1) }\end{array}$ & $\begin{array}{l}\text { Haemaphysalis } \\
\text { punctata (1), } \\
\text { Ixodes } \\
\text { trianguliceps } \\
\text { (1), Ixodes sp. } \\
\text { (4) }\end{array}$ & - \\
\hline & $\begin{array}{l}\text { No. of rodents infected } \\
\text { by flea: } 16\end{array}$ & $\begin{array}{l}\text { No. of rodents } \\
\text { infected by louse: } 13\end{array}$ & $\begin{array}{l}\text { No. of rodents } \\
\text { infected by } \\
\text { tick: } 6\end{array}$ & $\begin{array}{l}\text { No. of rodents } \\
\text { infected by } \\
\text { mite: } 0\end{array}$ \\
\hline \multirow[t]{2}{*}{ Autumn } & $\begin{array}{l}\text { Nosopsylla fasciatus (6), } \\
\text { Nosopsyllus iranus (1), } \\
\text { Nosopsylla sp. (1), } \\
\text { Xenopsylla buxtoni (2) }\end{array}$ & $\begin{array}{l}\text { Hoplopleura captiosa } \\
\text { (3), Polyplax asiatica } \\
\text { (2), Polyplax gerbilli } \\
\text { (1) }\end{array}$ & $\begin{array}{l}\text { Haemaphysalis } \\
\text { punctata (3), } \\
\text { Haemaphysalis } \\
\text { sp. (1) }\end{array}$ & $\begin{array}{l}\text { Haemolaelaps } \\
\text { sp. (5), Laelaps } \\
\text { sp. (1), } \\
\text { Microtrombicula } \\
\text { sp. (1) }\end{array}$ \\
\hline & $\begin{array}{l}\text { No. of rodents infected } \\
\text { by flea: } 9\end{array}$ & $\begin{array}{l}\text { No. of rodents } \\
\text { infected by louse: } 5\end{array}$ & $\begin{array}{l}\text { No. of rodents } \\
\text { infected by } \\
\text { tick: } 4\end{array}$ & $\begin{array}{l}\text { No. of rodents } \\
\text { infected by } \\
\text { mite: } 7\end{array}$ \\
\hline \multirow[t]{2}{*}{ Winter } & $\begin{array}{l}\text { Nosopsylla fasciatus } \\
(10), \text { Nosopsyllus iranus } \\
(1), \text { Xenopsylla sp. (1) }\end{array}$ & $\begin{array}{l}\text { Hoplopleura captiosa } \\
\text { (4), Polyplax asiatica } \\
\text { (1), Polyplax gerbilli } \\
\text { (1), Polyplax } \\
\text { spinulosa (3) }\end{array}$ & $\begin{array}{l}\text { Haemaphysalis } \\
\text { sp. (1), Ixodes } \\
\text { trianguliceps } \\
(2)\end{array}$ & $\begin{array}{l}\text { Hirstionyssus } \\
\text { sp. (1), Laelaps } \\
\text { sp. (1) }\end{array}$ \\
\hline & $\begin{array}{l}\text { No. of rodents infected } \\
\text { by flea: } 13\end{array}$ & $\begin{array}{l}\text { No. of rodents } \\
\text { infected by louse: } 11\end{array}$ & $\begin{array}{l}\text { No. of rodents } \\
\text { infected by } \\
\text { tick: } 3\end{array}$ & $\begin{array}{l}\text { No. of rodents } \\
\text { infected by } \\
\text { mite: } 2\end{array}$ \\
\hline
\end{tabular}

\section{Parasitism interaction and host sexuality}

Distribution of ectoparasite was also affected by host sex which is called sex-biased parasitism. Overall prevalence of four groups of ectoparasite on male rodents in comparison with females was recorded (56.4\% vs. $44.4 \%)$. However, similar mean intensity values were detected for both sexes (1.21 in males and 1.28 in females). Flea Nosopsyllus fasciatus, louse Polyplax asiatica and ticks of genus Haemaphysalis 
were the most abundant ectoparasite, among other members of their relevant group, in both male and female rodents. Although genus Haemolaelaps was the most abundant mite parasitized male hosts, but members of two genera Haemolaelaps and Laelaps showed equal abundance on females.

Host age is another trait which could have effect on the ectoparasite assemblage parasitizing hosts. Overall prevalence of four groups of ectoparasite on mature hosts was greater in comparison with immature ones (approximately 51\% vs. 47\%). In contrast, the mean intensity values of 1 and 1.34 were recorded for mature and immature rodent hosts, respectively (Table 6).

Table 6

Age-biased parasitism of rodents host harboring different groups of ectoparasite in northeastern Iran

\begin{tabular}{|lllll|}
\hline Host age & $\begin{array}{l}\text { No. of captured } \\
\text { rodent }\end{array}$ & $\begin{array}{l}\text { No. of infected } \\
\text { rodent }\end{array}$ & $\begin{array}{l}\text { Prevalence of } \\
\text { infection }\end{array}$ & $\begin{array}{l}\text { Mean intensity of } \\
\text { infection }\end{array}$ \\
\hline Immature & 49 & 23 & $46.9 \%$ & 1.34 \\
\hline Mature & 235 & 120 & $51 \%$ & 1 \\
\hline Total & 284 & 143 & & \\
\hline
\end{tabular}

Manifestation of sexual differences in ectoparasite infestation was different among male and female hosts between seasons. In spring, the prevalence of overall infection for males and females were $65.4 \%$ and $57.1 \%$, respectively. Likewise, nearly similar values of prevalence were recorded for males $(44.1 \%)$ and females (44\%) in winter. In contrast, male-biased parasitism was found in summer and autumn; prevalence in males was at about four times the value recorded for females (74.1\% vs. $17.5 \%)$ in summer, and during the autumn, this was about 1.5 times ( $60 \%$ vs. $36.6 \%)$.

Among all sampling localities, the highest prevalence (100\%) was recorded for Robat-e mahi (Razavi Khorasan province), Faruj, Bojnourd, Shirvan, Shirvan-Bojnourd road, and Siman Factory (North Khorasan province), and Qaen, Birjand, Sarayan, Nehbandan, Ferdows (South Khorasan province). In contrast, the lowest prevalence with the value of zero was recorded for Baba Ramezan, Chenaran, Golbahar, Kahoo, and Tirgan in Razavi Khorasan province, and Shadan and Olang heights in South Khorasan province.

Among habitats which monitored for capturing rodents, semi-deserts, followed by farms and gardens, and also rocky areas showed the highest prevalence of ectoparasites $(66.6 \%, 62.5 \%$, and $61 \%$, respectively). The lowest prevalence was recorded for the forest, may be due to sampling error and capturing no rodents there. The mean intensity of the flea infection was highest in the rocky areas (0.74), grasslands (0.63), and farms and gardens (0.6), while louse infection showed the highest value for mean intensity in meadows (0.66), sandy soils (0.66), and public areas (0.6). Same mean intensity of infection of flea and louse was reported from parks and semi-deserts (0.5), but woodlands had same intensity for flea and tick (0.5).

\section{Discussion}


In the present study the role of host traits (sex and age as biotic factors), as well as season and habitat (as abiotic factors) on parasitism interactions of the rodents of northeastern Iran were documented. Complex interactions between the host and its parasites as well as the co-existence among different groups of parasites resulted in the occurrence of a particular parasite species living on more than one host species [46]. This may be related to the intra and interspecific relationships, behavior and the microhabitats utilized by the host [47].

Different habitats and ecological niches may harbor different taxonomic composition of host species (e.g. $[24,28,48])$. According to several studies, the level of habitat disturbance may be resulted in increased prevalence of hosts which subsequently may increase the intensity rate of infestation with ectoparasites. For example, Paramasvaran and colleagues [3] showed that Rattus rattus diardii from urban area in Malaysia had the most diverse assemblage of ectoparasites among all other studied rodents. Habitat disturbance may also change the structure of mammal communities which may induce parasite hostswitches [49]. High zoonotic risk may be observed in most parasites that have a broad host range due to their vectorial capacity for zoonotic pathogens [50]. For example, in spite of showing different levels of host specialization in ticks, from generalists to the most exclusive species-specific parasites, the majority of them select different groups of vertebrates as hosts at different stages of their life [51]. Thus, assessing host specificity and the pattern of host-parasite associations is important as it is related to management of zoonotic diseases.

Host-ectoparasite specificity can also be influenced by other factors such as distribution, ecology and habitat preference by the host. For example, tree shrews family Tupaiidae (order Scandentia) showed lower ectoparasite loads as compared with rodents of family Scuiridae and Muridae. This is probably due to the fact that their fur provides less optimal micro-habitat for ectoparasites. Additionally, special behavior of these species such as irregular usage of the nest (e.g. in large tree shrew Tupaia tana) may be considered as another probable reason [52].

Last, host conditions such as ecological and behavioral characteristics, access to feeding resources and burrow opportunities, living in colonies or solitary, diversity and number of predators, also morphological features such as relative size and differences in the skin and its covering, as well as physiological factors such as difference in blood hormonal levels due to stress, differences in geographic and environmental factors, and nowadays, global warming have effects on differences in parasitism patterns and interactions between the hosts and their parasites [53]. All in all, although numerous studies on hostparasite associations have been carried on and detailed descriptions of the main groups of ectoparasites can be found elsewhere, but it still is difficult to propose general ecological patterns and rules about ectoparasite ecology and communities $[54,55]$.

In the present study, Meriones persicus was the most captured rodent species which inhabits rocky areas. Mus muculus as the most common rodents found in public areas, took the second place. This is in accordance with author's previous study showing higher total prevalence rate of ectoparasites in Mus rather than in Apodemus and Nesokia [28], likely due to nesting in habitats commensal with humans. This 
provides benefits like offering potentially rich food resources, which resulted in increased densities of the species and subsequent increase in prevalence and general index of its ectoparasites $[11,12]$.

With regards to the fact that the most diverse ectoparasite assemblage with moderate mean intensity were recorded for these two hosts, they are considered as a critical concern threatened their coexisting animals including humans. As mentioned earlier, the greatest number of captured rodents were infested by only one group of ectoparsites, and the most diverse ectoparasitic group with highest prevalence and infestation rate was fleas followed by lice, among which the most dominant species were flea Nosopsyllus fasciatus and louse Polyplax asiatica. Nosopsyllus fasciatus and Polyplax asiatica, exhibiting low and moderate host specificity, respectively, can be found all round the year (with highest mean intensity in winter) on both male and female hosts in most of the sampling localities and habitats. These all, may increase the probability of transferring infestation from their hosts to the other coexisting rodents' species.

The northern rat flea, Nosopsyllus fasciatus, is considered as a cosmopolitan species which hosted several numbers of rodent species including Arvicola terrestris, Apodemus sylvaticus, Cricetutus migratorius, Meriones libycus, Meriones persicus, Mesocricetus auratus, Microtus paradoxus, Microtus socialis, Mus musculus, Nesokia indica, Rattus norvegicus, Rattus rattus, Rhombomys opimus, Scarturus williamsi, and Spermophilus fulvus [22]. Rats are the primary hosts for this flea species but it also feeds on humans when necessary which causes irritation and swelling following flea bite. This flea can be vector of Yersinia pestis as plague bacteria. Plague disease appears with special signs and symptoms including painful enlarged lymph glands which is called a "bubo", fever, chills, and prostration in case of bubonic plague (inguinal bubo); chills, fever, diarrhea, abdominal pain, generalized pain, shock, arterial hypotension, rapid pulse, bleeding into skin and other organs, anxiety, slurred speech, mental confusion, prostration in septicemic plague; and cough, difficulty in breathing, chills, fever, rapid shock and death (if not treated early) in pneumonic plaque. Cool temperatures can facilitate transmission of this pathogen. Fatality rate of about $50-60 \%$ occurs in untreated bubonic plague. This flea species can spread other human diseases such as sleeping sickness caused by Trypanosoma sp. and salmonellosis caused by Salmonella sp. [56, 57].

The rat lice, Polyplax asiatica, which is only found in Asia, can parasitize the soricid species Suncus murinus as the type host and rodent species Bandicota bengalensis, Bandicota indica, Nesokia indica, Rattus pyctoris and Rattus rattus as the principal hosts. This murine lice species has been also recorded from Cricetulus migratorius, Meriones persicus, Spermophilus fulvus, and Tatera indica [22]. Generally, infestation with lice usually results in pruritus or itch, cutaneous inflammatory lesion, restlessness, debilitation, and sometimes death of host in heavy infestation. This lice, similarly to its congeneric members, do not parasitize man but can serve as vector for bacteria species Rickettsia prowazeki, which causes louse-borne typhus or epidemic typhus fever. Signs and symptoms of the disease may include fever and chills, headache, cough, rapid breathing, nausea, vomiting, body and muscle aches, rash, and confusion. Epidemic typhus is spread to people through contact with infected body lice. Although epidemic typhus was responsible for millions of deaths in previous centuries, it is now considered a rare disease [58]. 
Gender differences in ectoparasite infestation was observed in this study; males of captured rodents showed higher prevalence than females (79 vs 64 infested rodents out of 284 captured rodents). Higher prevalence was also recorded for mature rodents as compared with immature ones (120 vs 23 infested rodents out of 284 captured rodents). Generally, in most of the rodents species captured during this study, males were more inquisitive than the females, especially in spring (author's observations). This may have resulted from increased mobility of males during the mating season in order to locate females in estrus. However, a shift could be observed during late spring in which females might show greater mobility [59, 60]. This result can probably be explained by an increase in female's mobility, as they need to find favorable nesting areas and enough food for milk production during the reproductive season [61].

In the current study, higher intensity rate of infestation by flea was observed for females (1.09 vs 1.02). Some earlier studies indicated that male hosts are more seriously infested by fleas (e.g. [30]: house mouse Mus musculus), while others specified that females have higher infestation (e.g. [12]: commensal rats). In contrast, our results demonstrated that male hosts showed higher mean intensity by lice than females (1.39 vs 1.25). Some investigations recorded similarities of infestations by lice on female and male hosts (e.g. [62]: water rat Scapteromys aquaticus, [30]: Mus musculus), whereas others reported male-bias (e.g. [63]; four-striped grass mouse Rhabdomys pumilio). Herein, for ticks and mites, both male and female rodents showed same value of intensity $(=1)$. In some studies on ticks of rodents, no significant difference between infestations in host sexes was detected (e.g. [26]: several forest rodents; [30]: Mus musculus) but in other research, male mice showed greater tick loads than females and analyses suggested that this sexbias was related to body mass as opposed to sex category (e.g. [64]: wood mice Apodemus sylvaticus). Moravvej and colleagues [30] showed that there is no difference between hosts sex for the mite Trombiculidae on Mus musculus, which is in congruence with our results. Likewise, other studies displayed substantial higher prevalence rate of Laelapidae on males ([65]: African ground squirrels genus Xerus; [30]: Mus musculus).

In the present study, mature hosts exhibited higher prevalence of infestation by ectoparasites than immature ones. In contrast, in some studies, differences observed in prevalence rates of immature and mature hosts was not significant (e.g. [30]: Mus musculus) or immature hosts demonstrated a higher prevalence of ectoparasites than matures ([66]: Apodemus flavicollis).

All groups of ectoparasites were recorded all-round the year, except for the mites, which is likely due to sampling bias, as much more effort is need for collecting very small-sized mites on body of host. We found a clear trend in the seasonal distribution of the four common ectoparasite groups, in which fleas and lice, were more abundant in spring and winter, respectively. The higher number of records for ticks was in spring and summer and for mites this was in the autumn. This is in part, in accordance with previous records. Study on the relationship of ectoparasite prevalence of the murine rodent hosts to the capturing season in Razavi Khorasan province [28] demonstrated that lice and ticks had more abundant on murine species during cool wet months, whereas ticks and mites were more common during the hot dry months.

Topographic situation and climatic conditions in east of Iran, as one of the biodiversity hotspots [67], play an important role in distribution of rodents in this area and consequently their ectoparasitic fauna. Highest 
prevalence of infestation occurred in most of the sampling localities in North and South Khorasan provinces. On the other hand, the highest prevalence was observed in semi-desert biotope, which takes up most areas in these two provinces. Poor living conditions, poor and inadequate housing, lack of or little access to hygienic facilities, diet deficiency observed in some rural areas in North and South Khorasan provinces, as well as lack of sanitation and sufficient training programs in order to increase knowledge on rodents and parasites, precipitate spreading zoonotic diseases and the outbreak of pathogens of human health importance carried by infected animals. In addition, rodents in rural areas show larger home range and exhibited more movements, which resulted in increased possibility of being colonized by ectoparasites due to a greater chance of contact with individuals of the same and/or other species as compared with urban rodents [28].

In recent years, occurrence of zoonotic diseases has shown a great increase due to climatic change, deforestation, urbanization, and subsequent biodiversity loss. In this regard, near $75 \%$ of recently emerging diseases infected humans are diseases of animal origin [68]. Thus, first step to overcome these problems and preventing zoonoses emergence and development, is increasing the knowledge on rodents and their associated parasites and the way of the way of disease transmission and dealing with the probable pathogen through health monitoring program [69].

\section{Conclusions}

To conclude, the data presented in this study extend the knowledge on the distribution, seasonality and host choice of several members belonging to four main groups of ectoparasites in associations with several rodent species. Further studies are needed to can provide deep insight into how relationships and interactions between ectoparasite and rodents are formed, how they can be applied in predicting possible emergence of zoonotic diseases and analyzing the trend of epidemiology due to environmental changes resulted from anthropogenic activities.

\section{Declarations}

\section{Ethics approval and consent to participate}

Trapping and animal care was performed in compliance with the "Guidelines for the care and use of laboratory and experimental animals", Rodentology Research Group, Ferdowsi University of Mashhad. After ectoparasite removal, each rodent was released at its trapping point.

\section{Consent for publication}

Not applicable.

\section{Availability of data and materials}


The datasets used and/or analysed during the current study are available from the corresponding author on reasonable request.

\section{Competing interests}

The authors declare that they have no competing interests and have not a financial relationship with the organization that sponsored the research.

\section{Funding}

No funding sources.

\section{Authors' contributions}

$\mathrm{KH}$ initiated the study, carried out the rodent trapping, did the ectoparasites sample collection, identification and imaging, did the data analyses and wrote the first draft of manuscript. RBM made substantial contribution to revising the article critically for important intellectual content and had valuable discussions about the content. All authors read and approved the final manuscript.

\section{Acknowledgements}

The authors wish to thank A. Hamidi for his kind support and H. Mozaffari for field assistance.

\section{References}

1. Madinah A, Abang F, Mariana A, Abdullah MT, Mohd-Azlan J. Interaction of ectoparasites-small mammals in tropical rainforest of Malaysia. Community Ecol. 2014;15(1):113-20.

2. Krasnov BR, Morand S, Hawlena H, Khokhlova IS, Shenbrot GI. Sex-biased parasitism, seasonality and sexual size dimorphism in desert rodents. Oecologia. 2005;146(2):209-17.

3. Paramasvaran S, Sani RA, Hassan L, Krishnasamy M, Jeffery J, Oothuman P, et al. Ectoparasite fauna of rodents and shrews from four habitats in Kuala Lumpur and the states of Selangor and Negeri Sembilan, Malaysia and its public health significance. Trop Biomed. 2009;26(3):303-11.

4. Bradley CA, Altizer S. Urbanization and the ecology of wildlife diseases. Trends Ecol Evol. 2007;22(2):95-102.

5. Poláčiková Z. Ecology of mites (Acarina) on small mammals (Eulipotyphla, Rodentia) in Podunajská ní̌ina plain. Biologia. 2013;68(1):162-9.

6. Presley SJ. Interspecific aggregation of ectoparasites on bats: importance of hosts as habitats supersedes interspecific interactions. Oikos. 2011;120(6):832-41. 
7. Presley SJ. Sex-based population structure of ectoparasites from Neotropical bats. Biol J Linn Soc. 2012;107(1):56-66.

8. Sándor AD, Corduneanu A, Péter Á, Mihalca AD, Barti L, Csősz I, et al. Bats and ticks: host selection and seasonality of bat-specialist ticks in Eastern Europe. Parasite Vector. 2019;12(1):1-0.

9. Estrada-Peña A. Ticks as vectors: taxonomy, biology and ecology. Rev Sci Tech. 2015;34:53-65.

10. Morand S, De Bellocq JG, Stanko M, Miklisová YD. Is sex-biased ectoparasitism related to sexual size dimorphism in small mammals of Central Europe? Parasitology. 2004;129(4):505.

11. Soliman S, Main AJ, Marzouk AS, Montasser AA. Seasonal studies on commensal rats and their ectoparasites in a rural area of Egypt: the relationship of ectoparasites to the species, locality, and relative abundance of the host. J Parasitol. 2001;87(3):545-53.

12. Soliman S, Marzouk AS, Main AJ, Montasser AA. Effect of sex, size, and age of commensal rat hosts on the infestation parameters of their ectoparasites in a rural area of Egypt. J Parasitol. 2001;87(6):1308-16.

13. Rossin A, Malizia Al. Relationship between helminth parasites and demographic attributes of a population of the subterranean rodent Ctenomys talarum (Rodentia: Octodontidae). J Parasitol. 2002;88(6):1268-70.

14. Combes C. Parasitism. The ecology and evolution of intimate interactions. Chicago: University of Chicago Press; 2001.

15. Krasnov BR, Bordes F, Khokhlova IS, Morand S. Gender-biased parasitism in small mammals: patterns, mechanisms, consequences. Mammalia. 2012;76(1):1-13.

16. Lee CY, Alexander PS, Yang VV, Yu JY. Seasonal reproductive activity of male Formosan wood mice (Apodemus semotus): relationships to androgen levels. J Mammal. 2001;82(3):700-8.

17. Whiteman NK, Parker PG. Body condition and parasite load predict territory ownership in the Galapagos hawk. The Condor. 2004;106(4):915-21.

18. Moore SL, Wilson K. Parasites as a viability cost of sexual selection in natural populations of mammals. Science. 2002;297:2015-18.

19. Wells K, Lakim MB, Beaucournu JC. Host specificity and niche partitioning in flea-small mammal networks in Bornean rainforests. Med Vet Entomol. 2011;25(3):311-9.

20. Bittencourt EB, Rocha CF. Host-ectoparasite specificity in a small mammal community in an area of Atlantic Rain Forest (Ilha Grande, State of Rio de Janeiro), Southeastern Brazil. Mem. Inst. Oswaldo Cruz, Rio de Janeiro. 2003;98(6):793 - 98.

21. Darvish J, Rastegar-Pouyani E. Biodiversity conservation of reptiles and mammals in the Khorasan Provinces, Northeast of Iran. Progress Biol Sci. 2012;2(1):95-109.

22. Hamidi K, Nassirkhani M. Annotated checklist of fleas (Insecta: Siphonaptera) and lice (Insecta: Anoplura) associated with rodents in Iran, with new reports of fleas and lice. J Vector Dis. 2019;56(2):134-45.

23. Paulauskas A, Radzijevskaja J, Rosef O, Turčinavičiene J, Ambrasienė D. Infestation of mice and voles with Ixodes ricinus ticks in Lithuania and Norway. Est J Ecol Tallin: Estonian Academy of Sciences. 
2009;58(2):112-25.

24. Moravvej G, Hamidi K, Nourani L, Bannazade H. Occurrence of ectoparasitic arthropods (Siphonaptera, Acarina, and Anoplura) on rodents of Khorasan Razavi Province, northeast of Iran. Asian Pac J Trop. 2015;5(9):716-20.

25. Kaminskienė E, Radzijevskaja J, Balčiauskas L, Gedminas V, Paulauskas A. Laelapidae mites (Acari: Mesostigmata) infesting small rodents in the Curonian Spit. Lithuania Biologija. 2017;63(2):169-76.

26. Kiffner C, Vor T, Hagedorn P, Niedrig M, Rühe F. Factors affecting patterns of tick parasitism on forest rodents in tick-borne encephalitis risk areas, Germany. Parasitol Res. 2011;108(2):323-35.

27. Kiffner C, Stanko M, Morand S, Khokhlova IS, Shenbrot GI, Laudisoit A, Leirs H, Hawlena H, Krasnov BR. Variable effects of host characteristics on species richness of flea infracommunities in rodents from three continents. Parasitol Res. 2014;113(8):2777-88.

28. Hamidi K, Nourani L, Moravvej G. The relationship of ectoparasite prevalence to the capturing season, locality and species of the murine rodent hosts in Iran. Persian J Acarol. 2015;4(4):409-23.

29. Hamidi K, Nourani L, Moravvej G. New rodents' hosts of sucking lice, fleas (Insecta: Anoplura, Siphonaptera) and hard ticks (Acari: Ixodida) from Iran. Persian J Acarol. 2016;15(1):85-8. 5(.

30. Moravej G, Hamidi K, Nourani L. Relationship between the sex and age of Mus musculus (Rodentia: Muridae) with ectoparasites prevalence in northeast of Iran. PJA. 2016;5(1):51-62.

31. Kottek M, Grieser J, Beck C, Rudolf B, Rubel F. World map of the Köppen-Geiger climate classification updated. Meteorol Z. 2006;15(3):259-63.

32. Weather Atlas. Weather forecast and climate information for cities all over the globe. https://www.weather-atlas.com. Accessed 15 May 2020.

33. Islamic Republic of Iran Meteorological Organization Site. http://www.irimo.ir. Accessed 15 May 2020.

34. Heshmati GA. Vegetation characteristics of four ecological zones of Iran. Int J Plant Prod. 2012;1(2):215-24.

35. Fraser JL, Thompson GG, Moro D. Adequacy of terrestrial fauna surveys for the preparation of Environmental Impact Assessments in the mining industry of Western Australia. EMR. 2003;4(3):18792.

36. Corbet GB. The mammals of the Palaearctic region: A taxonomic review. London: British Museum (Natural History); 1978. p. 314.

37. Darvish J. Methodology in animal biosystematics. Mashhad: Jehad Daneshgahi Mashhad (JDM) Press; 2015.

38. Durden LA, Musser GG. The sucking lice (Insecta, Anoplura) of the world: A taxonomic checklist with records of mammalian hosts and geographical distributions. Bull Amer Museum Nat His; 1994.

39. Durden L, Kollars JT. The fleas (Siphonaptera) of Tennessee. J Vector Ecol. 1997;22(1):13-22.

40. Manilla G. Fauna D'Italia. Acari: Ixodida. Edizioni Calderini, Bologna Meerburg BG, Singleton GR, Kijlstra A. (2009) Rodent-borne diseases and their risks for public health. Crit Rev Microbiol. 1998;35:221 - 70. 
41. Baker AS. Mites and ticks of domestic animals: An identification guide and information source. The Stationary Office; 1999. p. 240.

42. Mašán P, Fend'a P. A Review of the Laelapid Mites Associated with Terrestrial Mammals in Slovakia, with a Key to the European Species (Acari: Mesostigmata: Dermanyssoidea). Bratislava: Institute of Zoology, Slovak Academy of Sciences; 2010.

43. Bush AO, Lafferty KD, Lotz JM, Shostak AW. Parasitology meets ecology on its own terms: Margolis et al. revisited J Parasitol. 1997;1:575-83.

44. Lareschi M, Notarnicola J, Navone G, Linardi PM. Arthropod and filarioid parasites associated with wild rodents in the northeast marshes of Buenos Aires, Argentina. Mem Inst Oswaldo Cruz. 2003;98(5):673-7.

45. Nava S, Guglielmone AA. A meta-analysis of host specificity in Neotropical hard ticks (Acari: Ixodidae). Bull Entomol Res. 2013;103(2):216.

46. Thanee N, Kupittayanant S, Pinmongkholgul S. Prevalence of ectoparasites and blood parasites in small mammals at Sakaerat Environmental Research Station, Thailand. Thai J Agricul Sci. 2009;42(3):149-58.

47. Bittencourt EB, Rocha CF. Host-ectoparasite specificity in a small mammal community in an area of Atlantic Rain Forest (Ilha Grande, State of Rio de Janeiro), Southeastern Brazil. Mem. Inst. Oswaldo Cruz, Rio de Janeiro. 2003;98(6):793-8.

48. Lit E, Idris MI, Marni W, Mortada MJ, Abdullah MT. Species diversity of small mammals in Lubuk Sembilang Recreational Park, Langkawi Island, Kedah. In: Mokhtar M, Halim AS, editors RIMBA 2: Regional Sustainable Development in Malaysia and Australia. Universiti Kebangsaan Malaysia, Universiti Malaysia Sarawak and Charles Darwin University. Institute for Environment and Development (Lestari) and Universiti Kebangsaan Malaysia. 2012. p. 124-35.

49. Gettinger D, Ernest KA. Small-mammal community structure and the specificity of ectoparasite associations in central Brazil. Rev Brasileira de Biol. 1995;55:331-41.

50. Woolhouse MEJ, Gowtage-Sequeria S. Host range and emerging and reemerging pathogens. Emerg Infect Dis. 2005;11:1842-7.

51. Nava S, Lareschi M, Voglino D. Interrelationship between ectoparasites and wild rodents from northeastern Buenos Aires Province, Argentina. Mem Inst Oswaldo Cruz. 2003;98(1):45-9.

52. Shabrina MS, Rafaee H. Ectoparasitic acari of small mammals from montane area of Cameron Highlands. Pahang J Wildl Parks. 1993;12:49-60.

53. Krasnov BR, Korallo-Vinarskaya NP, Vinarski MV, Shenbrot GI, Mouillot D, Poulin R. Searching for general patterns in parasite ecology: host identity versus environmental influence on gamasid mite assemblages in small mammals. Parasitology. 2008;135(2):229-42.

54. Korallo NP, Vinarski MV, Krasnov BR, Shenbrot GI, Mouillot D, Poulin R. Are there general rules governing parasite diversity? Small mammalian hosts and gamasid mite assemblages. Divers Distrib. 2007;13(3):353-60.

55. Poulin R. Are there general laws in parasite ecology? Parasitology. 2007;134:763-76. 
56. Jefferson T, Demicheli V, Pratt M. Vaccines for preventing plague. Cochrane Database Syst Rev 2000; (2): CD000976.

57. Donnelly TM. Disease problems of small rodents. Ferrets, rabbits, and rodents: clinical medicine and surgery. St. Louis: Elsevier/ Saunders; 2004. pp. 299-315.

58. Andersson JO, Andersson SG. A century of typhus, lice and Rickettsia. Res Microbiol. 2000;151(2):143-50.

59. Hamidi K, Darvish J, Matin M, Malikov M. V.G. A field behavioral study of Calomyscus elburzensis (Rodentia: Calomyscidae); the effects of sexual and seasonal variations in the trapping success. Acta Zool Bulgar. 2015;67(4):521-28.

60. Hamidi K, Darvish J, Matin MM. Potential use of brush-tailed mice for evolutionary developmental biology studies: Calomyscus elburzensis an appropriate rodent species. JWB. 2018;2(2):6-14.

61. Madikiza ZJ, Bertolino S, Baxter RM, Do Linh San E. Seasonal, sexual and age-related variations in the live-trapping success of woodland dormice Graphiurus murinus. Zool Stud. 2010;49(6):797-805.

62. Lareschi M. The relationship of sex and ectoparasite infestation in the water rat Scapteromys aquaticus (Rodentia: Cricetidae) in La Plata, Argentina. Revista de biología tropical. 2006;54(2):6739.

63. Krasnov BR, Matthee S. Spatial variation in gender-biased parasitism: host-related, parasite-related and environment-related effects. Parasitology. 2010;137(10):1527-36.

64. Harrison A, Scantlebury M, Montgomery WI. Body mass and sex-biased parasitism in wood mice Apodemus sy/vaticus. Oikos. 2010;119(7):1099-104.

65. Hillegass MA, Waterman JM, Roth JD. The influence of sex and sociality on parasite loads in an African ground squirrel. Behav Ecol. 2008;19(5):1006-11.

66. Benedek AM, Sibru I, Lazăr AN, Gheoca DA. Ecological aspects of ectoparasites' infestation in the yellow-necked mouse (Apodemus flavicollis: Rodentia, Muridae) from Transylvania (Romania). In: Marascu-Klein V, Panaitescu FV, Panaitescu M, editors. Proceedings of the 11th International Conference on Environment: Advances in Environment, Ecosystems and Sustainable Tourism, Brasov, Romania,2013. p. 197-202.

67. Misonne X. Analyse zoogéographique des mammifères de l'Iran. Thèse (Dissertation)-UCL-Université Catholique de Louvain, Belgium 1957; p. 157.

68. Bueno-Marí R. Animal health and zoonoses in the context of "One World, One Health" concept. J Etiol Anim Health. 2015;1(1):1-5.

69. Ambu S. Rodents and disease-the never ending problem. leJSME. 2014;8(1):1-2.

\section{Figures}




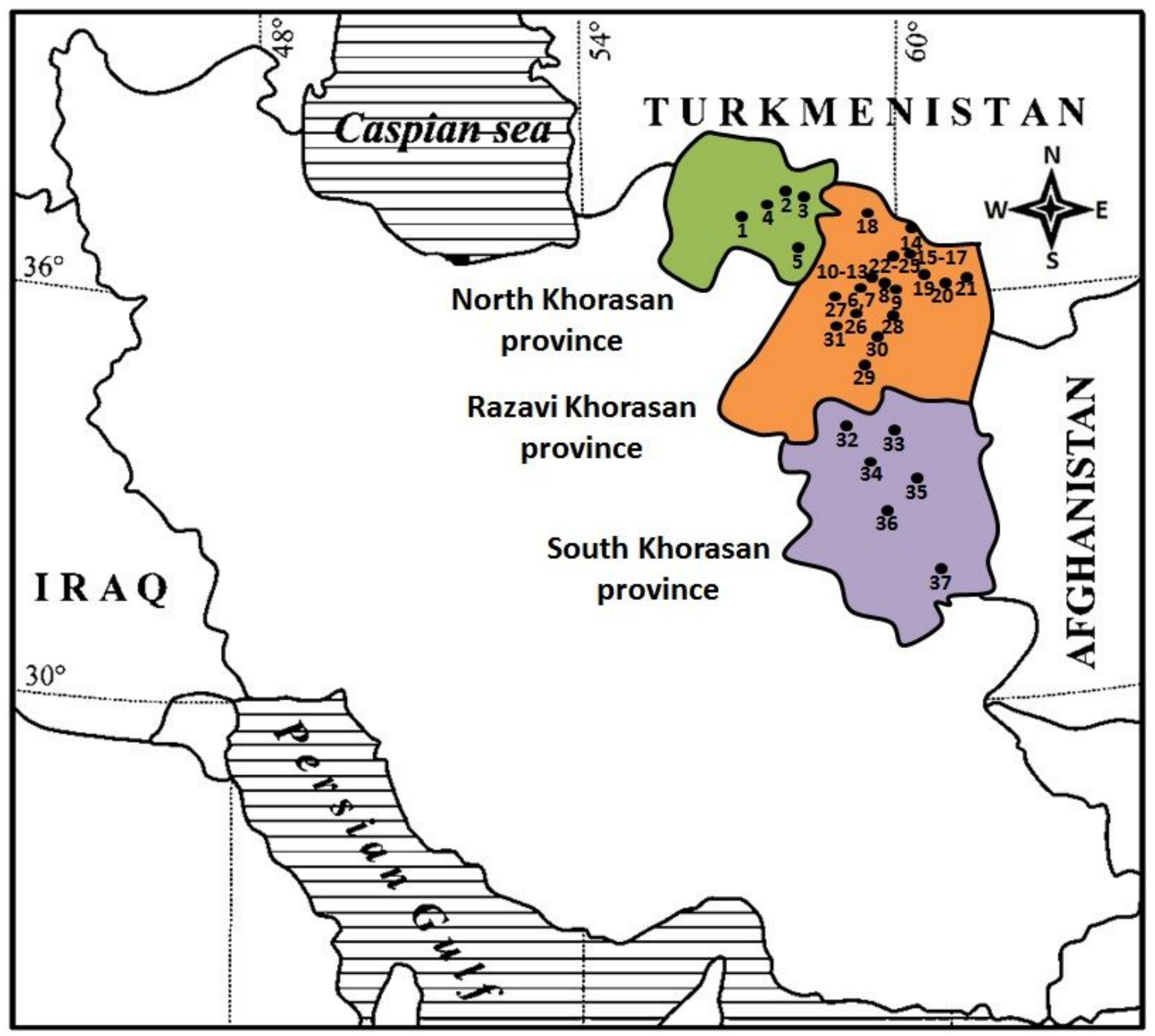

Figure 1

Geographical distribution of sampling locations for rodents in northeastern Iran. North Khorasan province: 1- Bojnourd, 2- Faruj, 3- Shirvan, 4- Siman factory,Shirvan, 5- Shirvan-Bojnourd road. Razavi Khorasan province: 6- Abardeh, Shandiz, 7- Cheshmegholgholi, Shandiz, 8- along the Torghabe river, Torghabe, 9Golbahar, 10- Chenaran, 11- Soltanabad, Chenaran, 12- Gorakhk, Chenaran, 13- Kahoo, Chenaran, 14- Baba Ramezan, Kalat, 15- Biuki, Kalat, 16- Pazh, Kalat, 17- Farmad, Kalat, 18- Tirgan, Dargaz,19- Robat-e mahi, Sarakhs, 20- Hasanabad, Sarakhs, 21- Chenarak, Sarakhs, 22- Firouzabad, Mashhad, 23- Hemmatabad, Mashhad, 24- Jimabad, Mashhad, 25- Manzelabad, Mashhad, 26- Hafthoz, Mashhad, 27- Khalaj, Mashhad, 28- Khaje-Morad, Mashhad, 29- Mashhad-TorbatHeydarieh road, 30-Sangbast, Mashhad-Torbat 
road,31- Dizbad, Neyshabur. South Khorasan province: 32- Qaen, 33- Ferdows, 34- Birjand, 35- Shadan and Olang heights, Birjand, 36- Nehbandan, 37- Sarayan.

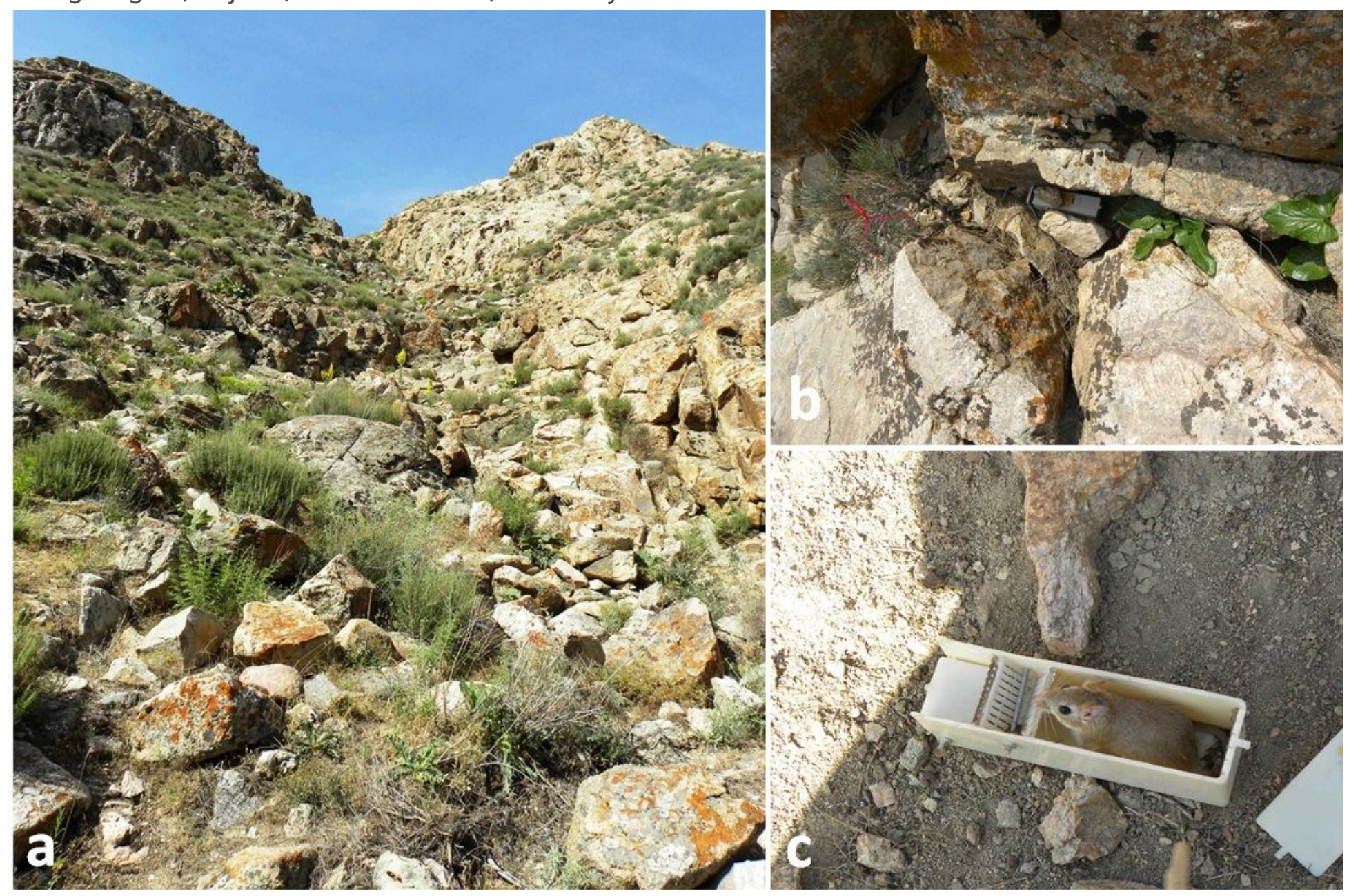

Figure 2

Sampling procedure for rodents. a Choosing a suitable habitat for the rodent species; Merionespersicus can be found in rocky areas of Khaje-Morad region, Mashhad, Razavi Khorasan province.b Finding a suitable point for placing traps; Merionespersicus generally uses rock cliffs and natural crevices as its nest. cReleasing the animal at its trapping pointafter ectoparasite removal. 


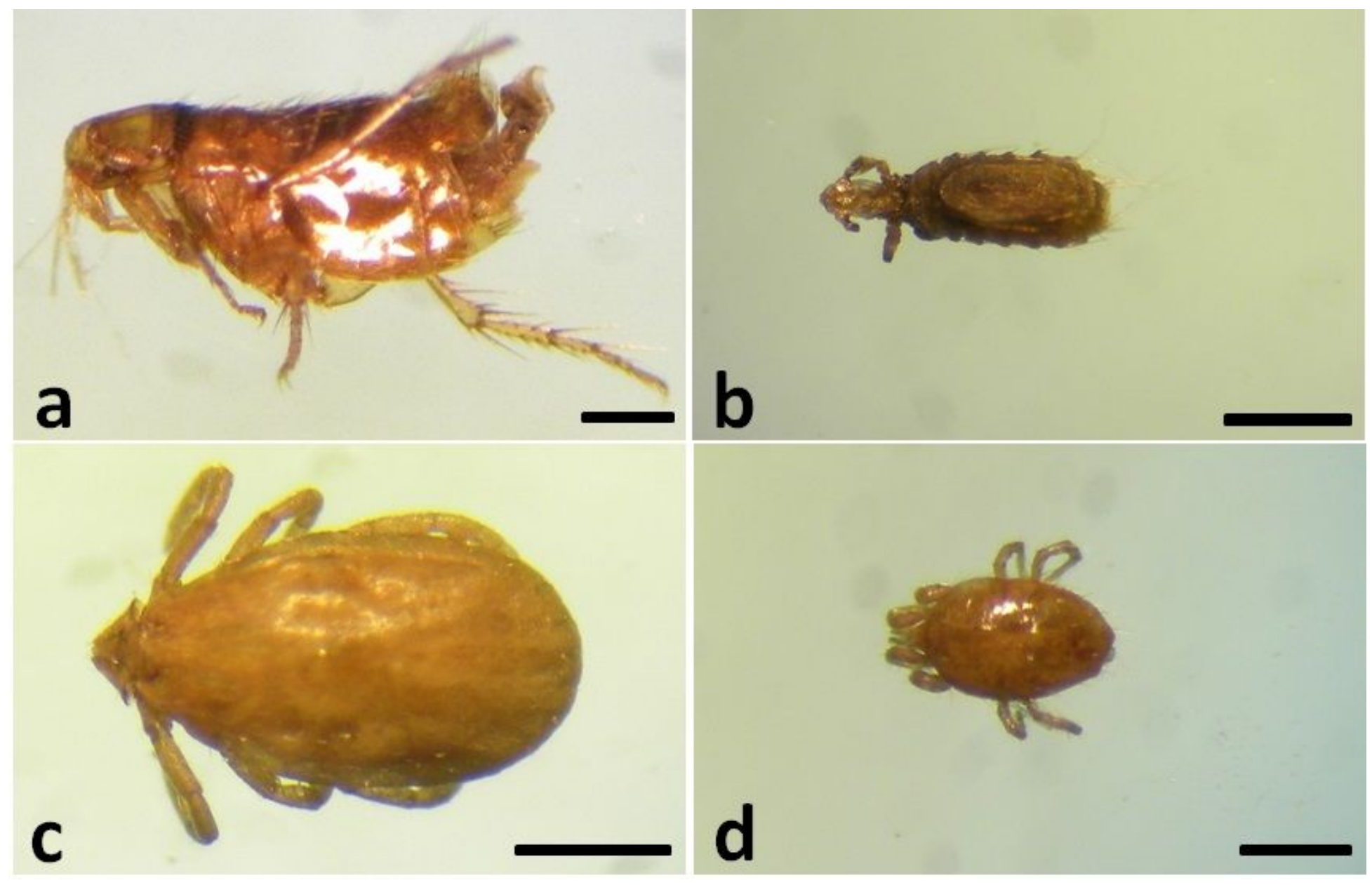

\section{Figure 3}

Collection of ectoparasite specimens found on the body surface of captured rodents. aFlea species Nosopsyllusfasciatus, blouse species Polyplaxasiatica, ctick species Haemaphysalispunctata, anddmite genus Haemolaelaps.Scale-bar: $0.5 \mathrm{~mm}$ 


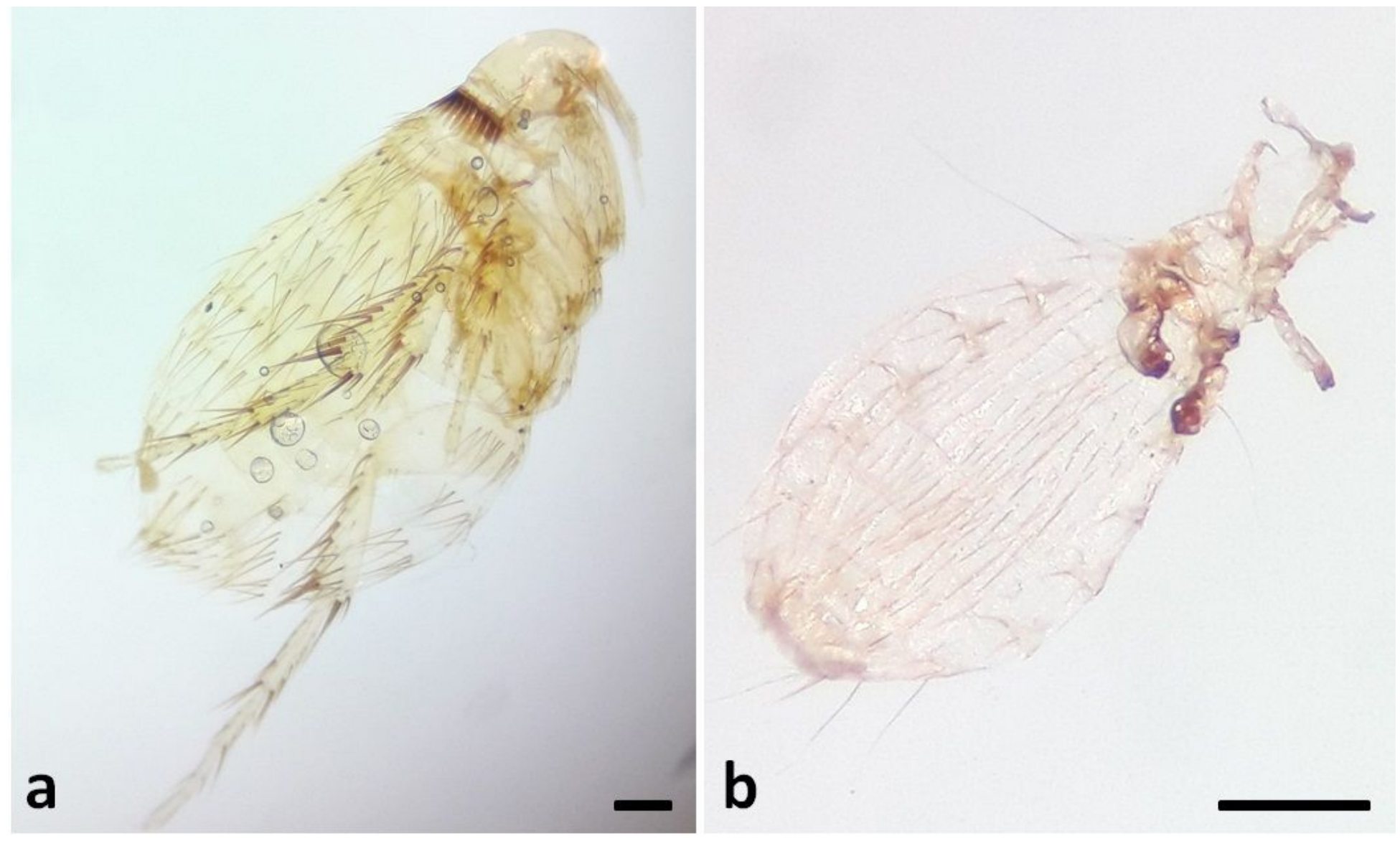

Figure 4

Stereoscopic imaging of ectoparasites after mounting clarified specimens in Hoyer medium. a)Flea species Nosopsyllusfasciatus, and b)louse species Polyplaxasiatica.Scale-bar: $250 \mu \mathrm{m}$ 